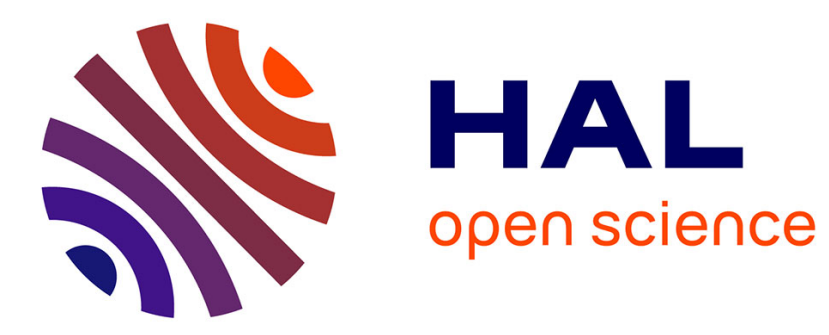

\title{
Species persistence decreases with habitat fragmentation: an analysis in periodic stochastic environments
}

\author{
Lionel Roques, Radu S. Stoica
}

\section{To cite this version:}

Lionel Roques, Radu S. Stoica. Species persistence decreases with habitat fragmentation: an analysis in periodic stochastic environments. 2007. hal-00149539

\section{HAL Id: hal-00149539 \\ https://hal.science/hal-00149539}

Preprint submitted on 28 May 2007

HAL is a multi-disciplinary open access archive for the deposit and dissemination of scientific research documents, whether they are published or not. The documents may come from teaching and research institutions in France or abroad, or from public or private research centers.
L'archive ouverte pluridisciplinaire HAL, est destinée au dépôt et à la diffusion de documents scientifiques de niveau recherche, publiés ou non, émanant des établissements d'enseignement et de recherche français ou étrangers, des laboratoires publics ou privés. 


\title{
Species persistence decreases with habitat fragmentation: an analysis in periodic stochastic environments
}

\author{
Lionel Roques Radu S. Stoica
}

\begin{abstract}
This paper presents a study of a nonlinear reaction-diffusion population model in fragmented environments. The model is set on $\mathbb{R}^{N}$, with periodic heterogeneous coefficients obtained using stochastic processes. Using a criterion of species persistence based on the notion of principal eigenvalue of an elliptic operator, we provided a precise numerical analysis of the interactions between habitat fragmentation and species persistence. The obtained results clearly indicated that species persistence strongly tends to decrease with habitat fragmentation. Moreover, comparing two stochastic models of landscape pattern generation, we observed that in addition to local fragmentation, a more global effect of the position of the habitat patches also influenced species persistence.
\end{abstract}

\section{Introduction}

These last 20 years, the number of species threatened by extinction has increased significantly. Among the many causes of threat, the first one for most of the endangered species is habitat destruction and associated fragmentation (see the Red List of Threatened Species [3]). Mathematical models incorporating habitat heterogeneities can be very helpful in understanding the links between the habitat fragmentation and the extinction processes. Since habitat fragmentation is in most cases the result of human activities, conservation measures derived from the observation of these models should be taken preventively.

There is a recent but abundant literature on population models taking account of habitat heterogeneities. The category of individual based models is represented by spatially explicit simulation models. In these models, where the landscape pattern is taken into account and each individual interacts with its environment, simulations are run until a general behaviour of the population can be observed. The advantages of these simulation models are that their implementation does not require sophisticated mathematical tools, and that they can incorporate precise information on the species behaviour. However, they remain limited in terms of general theoretical analysis, the number of parameters being quite high and the results of the simulations being closely linked 
to the choice of these parameters $[17,21,25]$. Conversely, diffusion models, although they sometimes make oversimplifying assumptions about movement of real organisms, provide an excellent tool for obtaining nontrivial theoretical and qualitative results on populations dynamics, which can lead to a better understanding of some ecological processes (see reviews in Murray [27], Okubo and Levin [28], Shigesada and Kawasaki [33], and Turchin [37]). In this paper, we choose to work with this last kind of model. More precisely, we modelled the species dynamics with a heterogeneous extension of the Kolmogorov equation, introduced simultaneously by Fisher [15] and Kolmogorov et al. [24]:

$$
\frac{\partial u}{\partial t}=D \nabla^{2} u+u(\mu(\mathbf{x})-\nu(\mathbf{x}) u), t \in \mathbb{R}, \mathbf{x} \in \mathbb{R}^{N}
$$

where $u=u(t, \mathbf{x})$ stands for the population density at time $t$ and position $\mathbf{x}$. This model is a combination of the diffusion equation, describing the motion of an important number of particles, each one following a random walk, and the logistic equation, $\mu(\mathbf{x})$ corresponding to the intrinsic growth rate of the species and $\nu(\mathbf{x})$ being a "susceptibility to crowding" coefficient. We placed ourselves in the particular case of the dimension $N=2$, and we assumed that the coefficients $\mu(\mathbf{x})=\mu\left(x_{1}, x_{2}\right)$ and $\nu(\mathbf{x})=\nu\left(x_{1}, x_{2}\right)$ were periodic, which means that there exist $L_{1}, L_{2}>0$ such that for all $\left(x_{1}, x_{2}\right)$ in $\mathbb{R}^{2}, \mu\left(x_{1}+L_{1}, x_{2}\right)=\mu\left(x_{1}, x_{2}+L_{2}\right)=$ $\mu\left(x_{1}, x_{2}\right)$ and $\nu\left(x_{1}+L_{1}, x_{2}\right)=\nu\left(x_{1}, x_{2}+L_{2}\right)=\nu\left(x_{1}, x_{2}\right)$. These "heterogeneous models" in periodic environments were first introduced by Shigesada et al. [34], and were more extensively studied in $[5,6,33]$. There are two major interests of considering models in periodic environments. The first one, which will not be exploited in this work, is that it allows the definition of some particular solutions, the "periodic travelling waves" (see [4, 6, 33]). The second interest is that it lessens the boundary effects, and is therefore very important when one wants to isolate the effects of fragmentation.

While studying these models in dimension 1, Shigesada and Kawasaki [33] empirically proved the key role played by the instability of the solution 0 of (1.1). They wrote "For the invasion to be successful (...), the population must increase when rare". This idea was then made precise in a rigorous framework and in any space dimension by Berestycki et al. [5]. Indeed, they proved that the instability of the state 0 was equivalent to $\lambda_{1}<0$, where $\lambda_{1}$ in the principal eigenvalue of a linear elliptic problem (see below for a precise definition). Furthermore, they showed that $\lambda_{1} \geq 0$ implies that the population density $u(t, \mathbf{x}) \rightarrow 0$ uniformly in $\mathbb{R}^{N}$ as $t \rightarrow \infty$, whereas $u(t, \mathbf{x})$ converges to a positive and periodic stationary state $p(\mathbf{x})$ if $\lambda_{1}<0$. It means that, whenever $\lambda_{1} \geq 0$, the population tends to extinction, whereas the case $\lambda_{1}<0$ corresponds to persistence. It is worth noting that the obtained necessary and sufficient condition for species persistence, $\lambda_{1}<0$, is amazingly simple compared to the various underlying mechanisms that condition survival or extinction. Moreover, this value can be numerically computed, using finite element methods (see e.g. [13]).

In the recent literature, this eigenvalue $\lambda_{1}$ has been shown to be linked, in some sense, to habitat fragmentation (see [5, 19, 20, 33] and Cantrell and Cosner [8]-[11]). In the present case of a periodic environment, the rearrangement of 
the habitat described in [5] decreased the value of $\lambda_{1}$, showing that the optimal configuration in terms of species persistence was connected and satisfied some symmetry properties (Figure 1). In the case of a habitat lying on a grid Roques and Hamel [31] numerically computed these optimal configurations, which were proved to depend on the habitat abundance and on the amplitude of the variations of the coefficient $\mu$ (for $N=2$ ). However, we do not know if there is a monotonic relation between $\lambda_{1}$ and the fragmentation of the habitat. In order to answer this question, we first had to give a precise definition of "habitat fragmentation", and to relate this definition with a measure of the habitat fragmentation. There exist several ways of defining it, and to obtain hypothetical habitat distribution corresponding to these definitions. Neutral landscape models, originally introduced by Gardner et al. [16] to generate spatial patterns in the absence of structuring processes, have now evolved and can include several constraints that account for habitat fragmentation [22]. This last kind of landscape model is often used as an input to spatially explicit simulation models (see e.g. $[29,38])$.

In this paper, we choose to define habitat fragmentation by using models inspired from statistical physics. These models are also neutral landscape models in the sense that they are stochastic models of landscape pattern, and the value (habitat or non-habitat in this paper) assigned to a position $\xi$ in the pattern is a random variable [22]. Moreover, our models allow an exact control of the habitat abundance. This is really important for our study, since we want to isolate the effects of fragmentation.

The topology of the generated landscape patterns is controlled by the parameters of the corresponding stochastic models. Our aim was to get an insight into the way $\lambda_{1}$ varies with the landscape pattern topology, hence with these parameters. Since these landscape models are stochastic, for a fixed value of the parameters, many different patterns can be generated. Thus, another question was whether the standard deviation of $\lambda_{1}$, on habitats generated with the same value of the parameters, was high or not. This standard deviation controls the range of values taken by $\lambda_{1}$ for a fixed degree of habitat fragmentation.

In the next section of this paper, the rigorous definition of $\lambda_{1}$ as well as some useful mathematical results are recalled. In section 3 , models for the generation of fragmented habitats are presented. The first landscape model only takes account of the local interactions between the habitat patches, while the second one incorporates more global interactions. The value of $\lambda_{1}$ is numerically computed on patterns generated for different values of the landscape models parameters. The results, indicating a strong monotonic relation between $\lambda_{1}$ and the models parameters, are presented in section 4 . These results are further discussed in section 5 .

\section{Mathematical background}

Although the following definitions and results are still true in dimensions higher than 2 , and since our numerical computations are made in dimension 2 , for the 


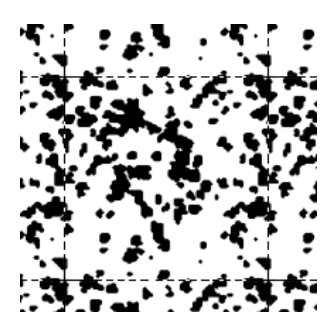

(a)

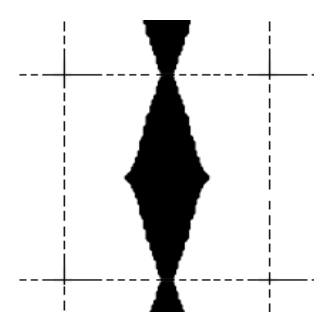

(b)

Figure 1: It was proved in [5] that, while having the same amount of habitat, the rearranged configuration (b) has always smaller values of $\lambda_{1}$ than the configuration (a). However, there was no result about the intermediate cases between (a) and (b).

sake of simplicity it is assumed in the sequel that $N=2$.

Definition of the eigenvalue $\lambda_{1}$

The operator $\nabla^{2}$ in equation (1.1) corresponds to the Laplace operator and is defined by $\nabla^{2} u=\frac{\partial^{2} u}{\partial x_{1}^{2}}+\frac{\partial^{2} u}{\partial x_{2}^{2}}$.

The positive stationary solutions $p(\mathbf{x})$ of (1.1) (i.e. the solutions that do not depend on the time variable $t$ ) are solutions of the following problem:

$$
\left\{\begin{array}{l}
-D \nabla^{2} p-p(\mu(\mathbf{x})-\nu(\mathbf{x}) p)=0, x \in \mathbb{R}^{2}, \\
p>0
\end{array}\right.
$$

The functions $\mu(\mathbf{x})$ and $\nu(\mathbf{x})$ are assumed to be periodic with the same period cell,

$$
C:=\left[0, L_{1}\right] \times\left[0, L_{2}\right],
$$

as explained in the introduction. We say that these functions are $L$-periodic. Moreover, it is assumed that $\mu, \nu \in L^{\infty}\left(\mathbb{R}^{2}\right)$. Under these conditions, the principal eigenvalue $\lambda_{1}$ of the linearized problem at 0 , associated with (2.2) is defined as the unique real number such that there exists a positive function $\Phi$ with

$$
\left\{\begin{array}{l}
-D \nabla^{2} \Phi-\mu(\mathbf{x}) \Phi=\lambda_{1} \Phi \text { in } \mathbb{R}^{2}, \\
\Phi \text { is L-periodic, } \\
\Phi>0 \text { and }\|\Phi\|_{\infty}=1 .
\end{array}\right.
$$

Existence and uniqueness of $\lambda_{1}$, as well as uniqueness of the function $\Phi$ satisfying these conditions follow from Krein-Rutman theory (see e.g. [2]). Moreover, since the elliptic operator of equation (2.3) is self-adjoint, $\lambda_{1}$ satisfies the following formula:

$$
\lambda_{1}=\min _{\Psi \in H_{p e r}^{1}, \Psi \neq 0} \frac{\int_{C} D|\nabla \Psi|^{2}-\mu(\mathbf{x}) \Psi^{2}}{\int_{C} \Psi^{2}},
$$


where $H_{\text {per }}^{1}$ is defined by $H_{\text {per }}^{1}=\left\{\Psi \in H_{\text {loc }}^{1}\left(\mathbb{R}^{2}\right)\right.$ such that $\Psi$ is L-periodic $\}$ $\left(\Psi \in H_{l o c}^{1}\left(\mathbb{R}^{2}\right)\right.$ means that for all compact subset $K$ of $\mathbb{R}^{2}, \Psi$ in $L^{2}(K)$, and the weak derivative of $\Psi$ is also in $L^{2}(K)$; see e.g. [1]).

\section{Necessary and sufficient condition of species persistence}

The condition $\lambda_{1}<0$ is necessary and sufficient for the existence of a bounded solution $p$ of (2.2). Moreover, when this solution exists, it is unique and $L$-periodic; these results are proved in [5]. Besides, as we said in the introduction of this paper, $\lambda_{1}<0$ is also a necessary and sufficient condition for the persistence of the modelled species, in the following sense: given an initial distribution of the species $u_{0}(\mathbf{x})$ at $t=0$ (being continuous nonnegative and bounded), if $\lambda_{1} \geq 0$ then the solution $u(t, \mathbf{x})$ of the model (1.1) converges uniformly in $\mathbb{R}^{2}$ to 0 . Thus the species tends to extinction. However, if $\lambda_{1}<0$, $u(t, x) \rightarrow p(\mathbf{x})$ uniformly on all compact set of $\mathbb{R}^{2}$. Since $p>0$, this can be considered as persistence (these results are demonstrated in [5]). Note that $\lambda_{1}$ does not depend on the function $\nu$ (it is in accordance with the previously mentioned citation of Shigesada and Kawasaki).

Moreover, as a consequence of the above uniqueness result, for all real $\alpha$, we have the following equation, which emphasizes the usefulness of minimizing $\lambda_{1}$ with respect to different arrangements of the function $\mu$,

$$
\lambda_{1}[\mu(\mathbf{x})-\alpha]=\lambda_{1}[\mu(\mathbf{x})]+\alpha .
$$

Indeed, formula (2.5) implies that the modelled species is all the more robust to a change in its growth rate as $\lambda_{1}[\mu]$ is small (other links between robustness to external perturbations and $\lambda_{1}[\mu]$ are discussed in [12]). Furthermore, it follows from (2.5) that $\lambda_{1}[\mu]$ being known, survival can easily be assessed for the whole family of growth rate functions $\{\mu(\mathbf{x})-\alpha\}_{\alpha \in \mathbb{R}}$.

\section{Stochastic models for habitat fragmentation}

The landscape models presented in this section to generate binary landscapes are described using Gibbs measures [36].

Here, we set $L_{1}=L_{2}=1$, thus $C=[0,1] \times[0,1]$. Let $C=\bigcup_{i=1}^{n_{C}} C_{i}$ be a finite partition of $C$, with $C_{i}$ equally sized disjoint square cells, and $n_{C}$ the number of cells. Let $\Xi$ be the lattice made of the centroids $\xi_{i}$ of the cells $C_{i}$, equipped with a neighbouring system $V_{\xi}$. Let $\mu^{+}$and $\mu^{-}$be two real numbers with $\mu^{+}>\mu^{-}$. Now, consider the random field $\omega$ defined on the lattice $\Xi$. This random field assigns each site $\omega_{\xi}(\xi \in \Xi)$ either the value $\mu^{+}$or $\mu^{-}$, while the number $n_{+}=\operatorname{card}\left\{\omega_{\xi}=\mu^{+}, \xi \in \Xi\right\}$, takes a pre-fixed value. Under these assumptions let $\Omega$ be the corresponding state space.

For each element $\omega \in \Omega$ the growth rate function $\mu$ on $C$ is defined by $\mu(\mathbf{x})=\omega_{\xi}$ whenever $\mathbf{x}$ belongs to $C_{\xi}$. This function is extended by periodicity to $\mathbb{R}^{2}$. The regions where $\mu(\mathbf{x})=\mu^{+}$are interpreted as "habitat" whereas the 


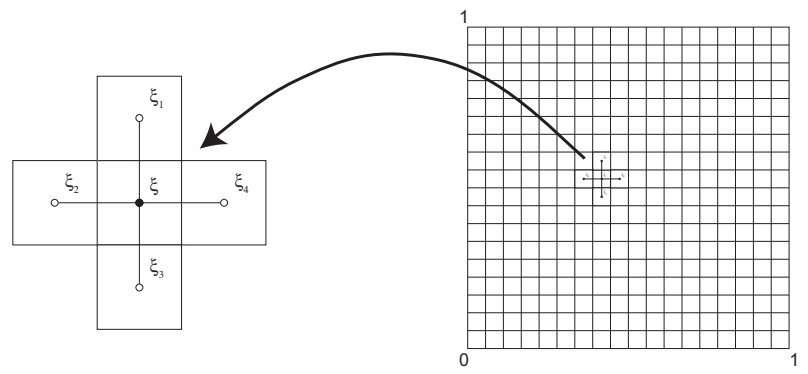

Figure 2: The 4-neighbourhood system: an element $\xi$ of $\Xi$ and its four neighbours $\xi_{i}, i=1$..4.

regions where $\mu(\mathbf{x})=\mu^{-}$correspond to "non-habitat". Thus, in the sequel, we shall talk of $\omega$ and the associated function $\mu$ as a landscape pattern.

In the following, we specify the neighbourhood system and the probability distribution of the defined random field. Our aim was to obtain models that simulate the landscape pattern, while its fragmentation was controlled by the models parameters.

Let $V_{\xi}$ be the 4-neighbourhood system as shown in Figure 2. In order to take account of the periodicity of the environment, the domain is considered wrapped on a torus.

For each $\omega$ in $\Omega$, we set $s(\omega)=\frac{1}{2} \sum_{\eta, \xi \in \Xi, \eta \in V_{\xi}} \mathbb{1}\left\{\omega_{\xi}=\omega_{\eta}\right\}$, the number of pairs of neighbours $\xi, \eta \in \Xi$ such that $\omega_{\xi}=\omega_{\eta}$ (11 $\{\cdot\}$ is the indicator function). The statistic of the model, $s(\omega)$, is directly linked to the habitat fragmentation: a landscape pattern is all the more aggregated as $s(\omega)$ is high, and all the more fragmented as $s(\omega)$ is small.

Landscape model 1

A first model for landscape generation, that we call landscape model 1 , is defined by the Gibbs measure $P$, defined over $\Omega$ by

$$
P(X=\omega)=\frac{1}{Z} e^{-U(\omega)},
$$

with the partition function $Z=\sum_{\omega \in \Omega} e^{-U(\omega)}$ and the Gibbs energy function $U$ : $\Omega \rightarrow \mathbb{R}$

$$
U(\omega)=-\beta s(\omega),
$$

with $\beta>0$; thus, the probability density becomes

$$
P(X=\omega)=\frac{1}{Z} e^{\beta s(\omega)} .
$$




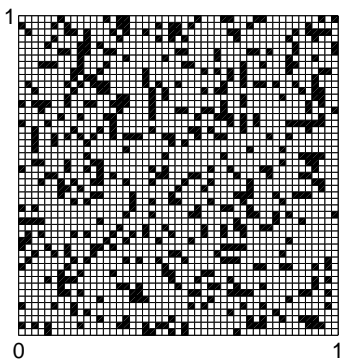

(a) $\beta=0.1$

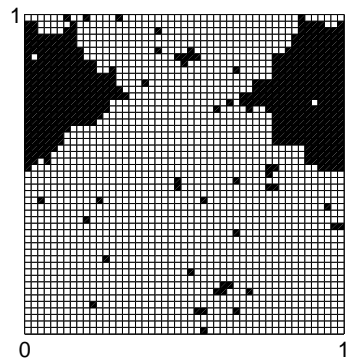

(d) $\beta=1.2$

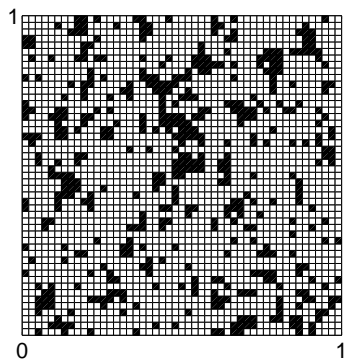

(b) $\beta=0.6$

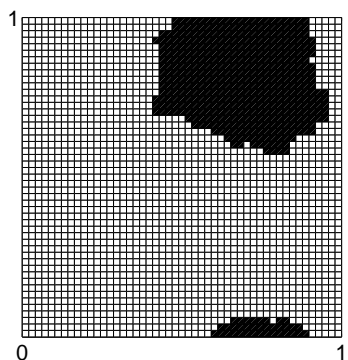

(e) $\beta=1.8$

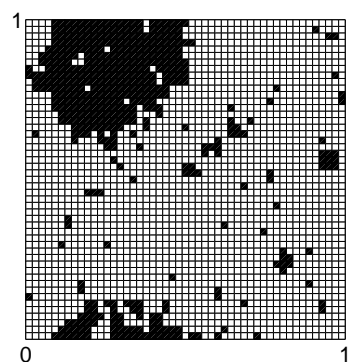

(c) $\beta=1$

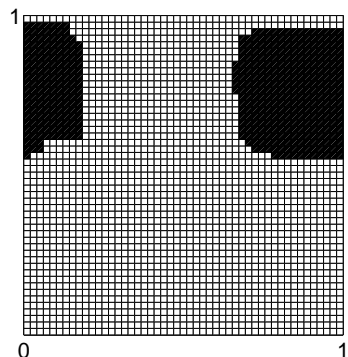

(f) $\beta=3$

Figure 3: Landscape model 1: obtained habitat patterns with different values of the parameter $\beta$. Habitat abundance has been set to $20 \%$.

The proposed landscape model favours the pairs of neighbours with the same value, while $\beta$ increases. Hence, $\beta$ directly controls the topology of the landscape patterns.

This probability distribution was simulated with a Metropolis-Hastings algorithm [18, 26]. The landscape samples were picked-up after running the Metropolis-Hastings dynamic a sufficient time, so that the stationarity of the Markov chain can be considered reached. It is worth noting that $\beta$ is the only "entry" parameter. Some samples are presented in Figure 3. As expected, we obtained all the more "aggregated" (as opposed to "fragmented") landscapes as the value of $\beta$ was high. This justifies the use of this model for the analysis of the variations of $\lambda_{1}$ with respect to the fragmentation of the landscape pattern. Another reason to vote for it is its simplicity because there is only one parameter, $\beta$.

For our numerical computation, fixed $n_{C}=50^{2}$ and the habitat abundance was set to $20 \%$, corresponding to $n_{+}=0.2 \times 50^{2}$.

Remark 3.1 The reader may note a similarity of the proposed landscape pattern model with the binary Ising model, since it uses "habitat" and "nonhabitat" states for the cells. However, in our case, there is an additional condition on the number of habitat cells. 


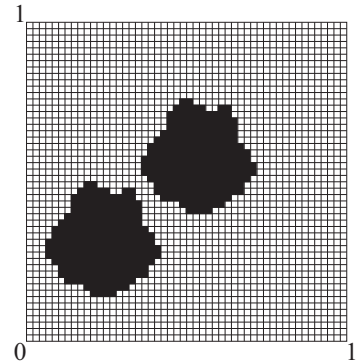

(a)

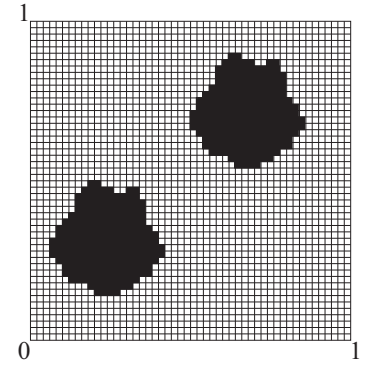

(b)

Figure 4: Two landscape patterns made of two identical components. In (a), the components are closer than in (b). The same energy is given by model 1 to both landscape patterns.

Remark 3.2 Under landscape model 1 assumptions, the energy (3.7) of the two landscapes of Figure 4 is the same, while, from a more realistic point of view (see e.g. [32]), we would like to differentiate between these two situations. The diffusive nature of the Laplace operator $\nabla^{2}$ also suggests that more global interactions between the landscape patches have to be taken into account in the definition of fragmentation. Intuitively, this should reduce the standard deviation of $\lambda_{1}$ for a fixed value of $\beta$.

\section{Landscape model 2}

As a consequence of Remark 3.2, we considered a second model, landscape model 2. A random point $\kappa$ is first chosen uniformly in the period cell $C$. Conditionally on $\kappa$, the Gibbs measure of the random field is

$$
P(X=\omega \mid \kappa)=\frac{1}{Z_{\kappa}} e^{-U_{\kappa}(\omega)},
$$

with $Z_{\kappa}=\sum_{\omega \in \Omega} e^{-U_{\kappa}(\omega)}$ and

$$
U_{\kappa}(\omega)=-\beta s(\omega)+\frac{1}{\sigma^{2}} \tau_{\kappa}(\omega)
$$

with $\sigma>0$ and

$$
\tau_{\kappa}(\omega)=\sum_{\eta \in \Xi, \omega_{\eta}=\mu^{+}} d(\eta, \kappa)^{2} .
$$

The function $d: C \times C \rightarrow \mathbb{R}$ is the distance over the "torus" $C$ defined by $d(\mathbf{x}, \mathbf{y})=\min _{\mathbf{z}=(i, j),-1 \leq i, j \leq 1}\|\mathbf{z}-\mathbf{x}+\mathbf{y}\|, \forall \mathbf{x}, \mathbf{y} \in C^{2}$

The additional term, $\frac{1}{\sigma^{2}} \tau_{\kappa}(\omega)$, compared to landscape model 1 , acts as an external field. This field has the shape of a 2-dimensional truncated Gaussian centered in $\kappa$ and with variance $\sigma^{2}$. We know that in our case, with a habitat 


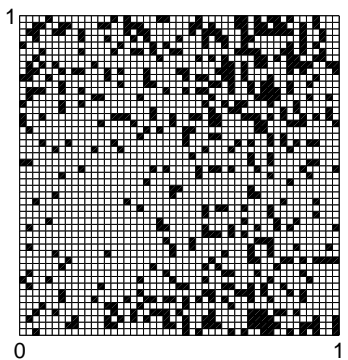

(a) $\beta=0.1$

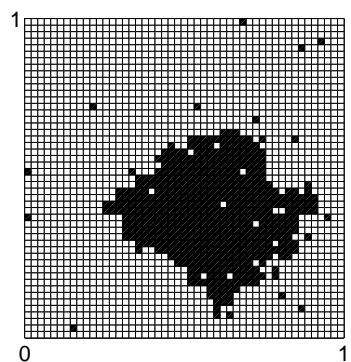

(d) $\beta=1$

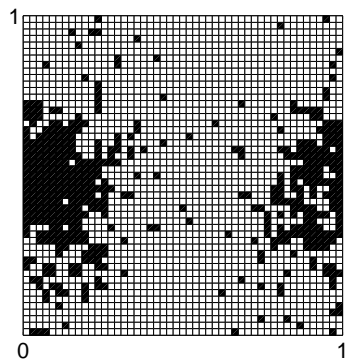

(b) $\beta=0.6$

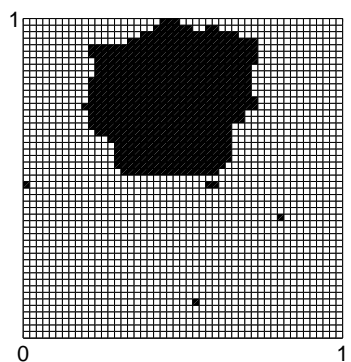

(e) $\beta=1.5$

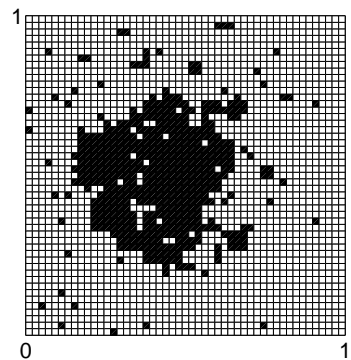

(c) $\beta=0.8$

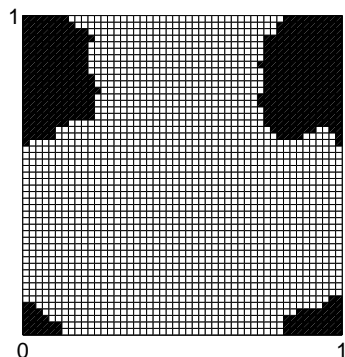

(f) $\beta=3$

Figure 5: Landscape model 2: generated habitat patterns with different values of the parameter $\beta$, with $\sigma=0.4$ and habitat abundance equal to $20 \%$.

abundance of $20 \%$, the optimal configuration of the environment is disc-shaped (at least when the amplitude $\left|\mu^{+}-\mu^{-}\right|$is not too small [31]). This was one of our motivations for the choice of such a "Gaussian" term. Samples of this new model are shown in Figure 5. The same values that in landscape model 1 were given to $n_{C}$ and $n_{+}$for the numerical computations.

\section{Simulation results}

This section is dedicated to the analysis of the variations of $\lambda_{1}$ with respect to the parameters of the two landscape models defined in the previous section.

The growth rate function was defined at the beginning of section 3. For our computations we set $\mu^{+}=7.8$ and $\mu^{-}=-2.2$ so that $\lambda_{1}$ can be positive or negative, depending on $\beta$. After several trials, the $\sigma$ value of landscape model 2 was set to 0.4 (indeed, if $\sigma$ is set to very low values the landscape patterns are too aggregated, independently of $\beta$; if $\sigma$ has too large values, the obtained model is equivalent to landscape model 1). Moreover, the diffusion coefficient was set to $D=1$.

For each element $\beta$ in the set $\{k \times 0.001, k=0 . .3000\}$, we generated 10 samples of each landscape model. The value of $\lambda_{1}$, satisfying equation (2.3) was computed on each sample using a finite element method (see e.g. [13]). The 


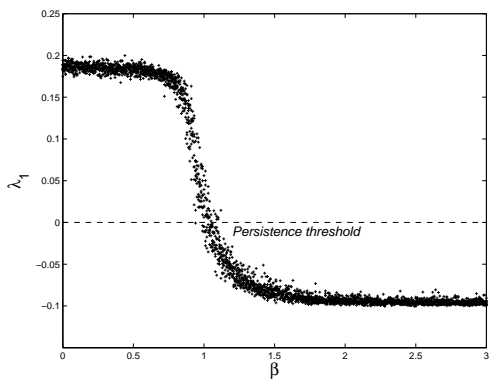

(a)

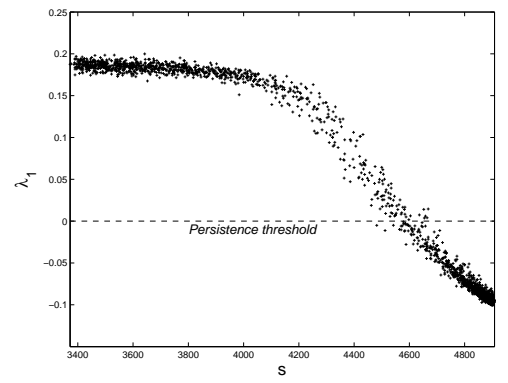

(b)

Figure 6: Landscape model 1: (a) dependency of $\lambda_{1}$ with respect to $\beta$, (b) scatter plot of the couples $\left(\lambda_{1}, s\right)$ for each generated landscape sample.

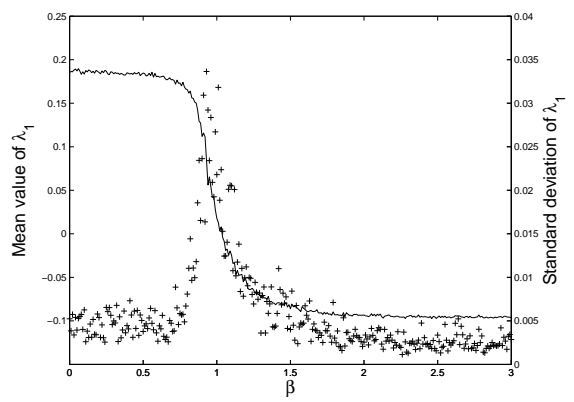

(a)

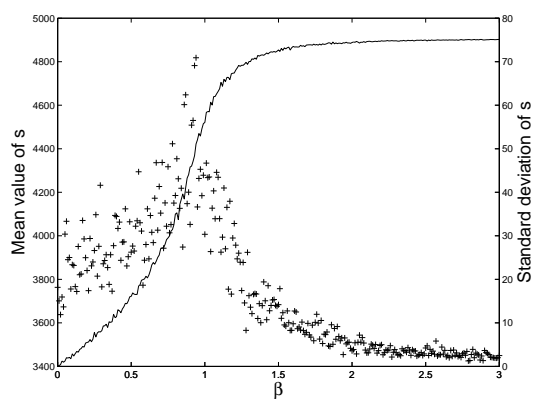

(b)

Figure 7: Landscape model 1: standard deviation (scatter plots) and mean value (solid lines) of the series of 10 computed values of (a) $\lambda_{1}$, in function of $\beta$; (b) $s$, in function of $\beta$.

statistic $s$ of each sample, defined in section 3, was also computed.

The results of the computations of $\lambda_{1}$, for landscape model 1 , are presented in Figure 6. A visual examination of the scatter plot of Figure 6 (a) reveals a monotonic tendency of the relationship between $\lambda_{1}$ and $\beta$. This tendency can be assessed by computing the Spearman correlation coefficient $\rho$. Whenever $\rho$ is -1 , this indicates a purely monotonic decreasing relationship (see Appendix A). However, since the landscape pattern used in the computation of $\lambda_{1}$ is a random variable, so is $\lambda_{1}$. This reason together with Remark 3.2 induce variability of $\lambda_{1}$ for fixed values of $\beta$. Therefore, what we expected from $\rho$ were rather negative values or in the best case values that were close to -1 . Such values were used to assess a monotonic behaviour of $\lambda_{1}$ with respect to $\beta$. An analysis over the entire range $\beta \in[0,3]$ gave $\rho=-0.93$. This indicates a highly decreasing behaviour of $\lambda_{1}$ with respect to $\beta$.

Figure 6 (a) suggests a deeper analysis by decomposing the $\beta$ domain. For $\beta \in[0,0.6]$, while the overall tendency is towards the aggregation, as assessed by 


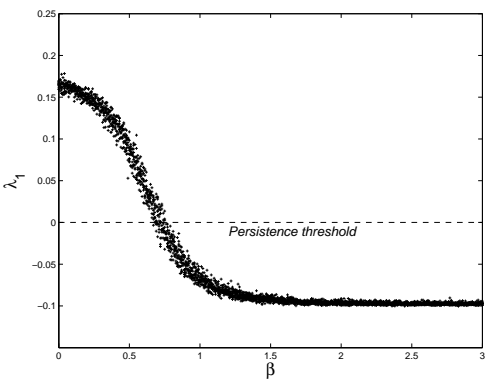

(a)

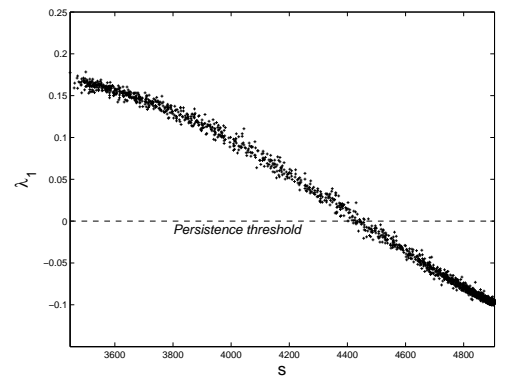

(b)

Figure 8: Landscape model 2: (a) dependency of $\lambda_{1}$ with respect to $\beta$, (b) scatter plot of the couples $\left(\lambda_{1}, s\right)$ for each generated landscape sample.

the mean value of $s$ (Figure $7(\mathrm{~b})$ ), the observed decreasing of $\lambda_{1}$ is slow. This is also indicated by $\rho=-0.28$. For $\beta \in[1.8,3]$, the fragmentation of the landscape varies very slowly, the obtained patterns being almost "aggregated" (Figure 7 (b)). This may explain the slow decreasing of $\lambda_{1}$ that can be noticed in Figure 6 (a), and indicated by $\rho=-0.31$. The case with $\beta \in[0.6,1.8]$ is the transition between the two situations described previously. As indicated by the mean value of $s$, the fragmentation of the landscape pattern varies strongly, between fragmented to aggregated patterns. This strong variation is also observed for the values of $\lambda_{1}$, and reflected by the corresponding $\rho=-0.97$.

A scatter plot of the couples $\left(\lambda_{1}, s\right)$ computed on each generated sample is also presented in Figure 6 (b), confirming the decreasing tendency of $\lambda_{1}$ as the aggregation of the habitat increases $(\rho=-0.94)$.

The dependency of $\lambda_{1}$ with respect to $\beta$ for landscape model 2 was analyzed for a fixed value of $\sigma$. The computation of Spearman's $\rho$ for the whole range $\beta \in[0,3]$ gave $\rho=-0.94$. The obtained results that are shown in Figure 8 (a) suggest an analysis on the two intervals $[0,1.5]$ and $[1.5,3]$. The corresponding Spearman's $\rho$ values are $\rho=-0.99$ and $\rho=-0.48$ respectively. As for landscape model 1 , a scatter plot of the couples $\left(\lambda_{1}, s\right)$ (Figure $8(\mathrm{~b})$ ) obtained on each sample confirms the decreasing tendency of $\lambda_{1}$, as the landscape becomes more aggregated, with $\rho=-0.94$.

The standard deviation of each series of 10 values of $\lambda_{1}$ for landscape model 1 is presented in Figure 7 and in Figure 9 for landscape model 2, together with the standard deviation of the corresponding series of the statistic $s$, for each $\beta$. For both models, the maximal standard deviation of $\lambda_{1}$ is encountered for the values of $\beta$ allowing a maximum variability of $s$. This maximal standard deviation is around 0.035 for landscape model 1 , and around 0.018 for landscape model 2. It remains quite small compared to the whole range of values taken by $\lambda_{1}$, $(-0.10,0.20)$ for landscape model 1 and $(-0.10,0.18)$ for model 2 , as $\beta$ varies. This means that, while for a fixed value of $\beta$, the topology of the generated landscape varies greatly, the computed values of $\lambda_{1}$ remains "stable". Moreover, 


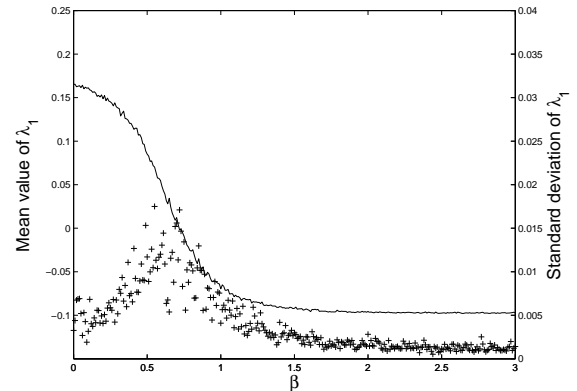

(a)

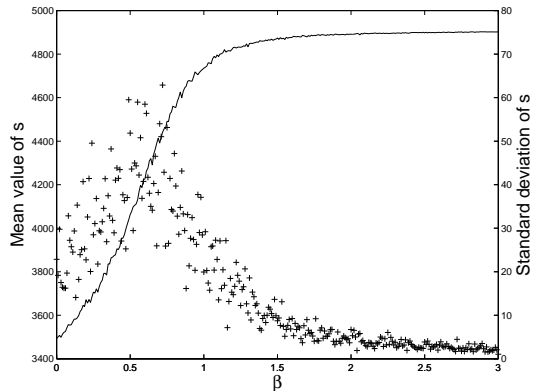

(b)

Figure 9: Landscape model 2: standard deviation (scatter plots) and mean value (solid lines) of (a) $\lambda_{1} ;$ (b) $s$.

it is also worth noting that although the standard deviations of the series of $s$ are comparable in landscape models 1 and 2, the corresponding maximal standard deviation of $\lambda_{1}$ for landscape model 2 is almost two times smaller. The Spearman coefficients, closer to -1 in landscape model 2 compared to landscape model 1 , may be the reflect of this lower standard deviation of $\lambda_{1}$. This lower standard deviation of $\lambda_{1}$ also supports the arguments of Remark 3.2.

Remark 4.1 In all the above situations, the obtained p-values were smaller than $10^{-10}$.

Remark 4.2 Note that some variations could also be caused by the numerical method used for computing $\lambda_{1}$. We used a sufficiently refined mesh to ensure that these variations remain small (of order $10^{-4}$ ). Besides, some variations in the dependency of $\lambda_{1}$ with respect to $\beta$ could also be the consequence of the way the landscape patterns were simulated, using a Metropolis-Hastings algorithm, for which convergence speed is not known [30].

\section{Discussion and conclusions}

Using a necessary and sufficient condition of species persistence in periodic heterogeneous environments, proved in [5], we analyzed the survival tendency (persistence or extinction) of species whose dynamics are modelled by the reactiondiffusion equation (1.1), in function of the habitat fragmentation.

Working in an infinite periodic environment allowed us to remove most of the boundary effects which are encountered with Dirichlet (lethal) or Neumann (no flux) boundary conditions. To our knowledge, such an approach is not possible with simulation models, for which the domain has to be finite. We were therefore able to focus on the sole effect of habitat fragmentation.

We considered two models for landscape generation. In both cases, the habitat abundance was fixed and the fragmentation was controlled via a common 
parameter, $\beta$. In the first landscape model, the generation of the patterns only took account of the number of pairs of similar neighbours, whereas in the second one, the proximity of habitat patches, even if they were not neighbours, was also taken into account. The stochastic nature of these models enabled us to explore a wide range of landscape patterns, allowing to obtain more general results than if we had analyzed a particular type of deterministic environment. Indeed, for a fixed value of the parameter $\beta$, the standard deviation of $\lambda_{1}$ remained low, with an advantage for landscape model 2. This indicates that, to each degree of fragmentation corresponds a narrow range of values $\lambda_{1}$. Thus, the fragmentation level being fixed by the parameter of our landscape models, the chances of persistence do not vary greatly with the topology of the landscape pattern.

Moreover, for both models, a strongly monotonic relation was found between their parameter $\beta$, and $\lambda_{1}$, while the habitat fragmentation was also strongly correlated to $\beta$. Among all the possible configurations of $\mu$, the more fragmented ones, obtained with landscape model 1 and $\beta$ close to 0 , were the worst in terms of species survival. Indeed, for small values of $\beta$, landscape model 1 gave values of $\lambda_{1}$ very close to 0.2 ( 0.18 for landscape model 2$)$, while 0.2 is precisely an upper bound of $\lambda_{1}[\mu]$. Indeed, from (2.4), since the constant function $\Psi \equiv 1$ belongs to $H_{p e r}^{1}$, we have $\lambda_{1} \leq-\frac{1}{|C|} \int_{C} \mu(\mathbf{x}) d \mathbf{x}$, thus, with a habitat abundance of $20 \%, C=[0,1]^{2}, \mu^{+}=7.8$ and $\mu^{-}=-2.2$, we get $\lambda_{1} \leq-0.2 \times 7.8+0.8 \times 2.2=$ 0.2 . On the contrary, the chances of persistence increase as the parameter $\beta$ and the associated aggregation of the habitat increase. These results support the idea of creating large reserves rather than small reserves in terms of species conservation.

In the landscape model 1 case, the relation between the number of pairs of similar neighbours $s$ and the value of $\lambda_{1}$ was clearly nonlinear (Figure $6(\mathrm{~b})$ ). The decreasing tendency of $\lambda_{1}$ was low for small values of $s$ and became more straightforward for higher values of $s$. From the biological conservation viewpoint, this means that a slight increase of the habitat aggregation has almost no effect on species persistence in the case of very fragmented landscapes. However, the persistence is improved by such a change in the case of more aggregated landscapes. For landscape model 2, the relation between $\lambda_{1}$ and $s$ was much closer to linear (Figure 8 (b)). Moreover, when the number of similar neighbours $s$ was low, the decreasing tendency of $\lambda_{1}$ with $s$ was faster than with landscape model 1 . This means that, in environments that are very fragmented (with low value of $s$ ) but when the habitat patches are close to each other, even if not neighbours, a small increase of the aggregation has a greater incidence on species survival.

The lower variability of $\lambda_{1}$ in landscape model 2 , for a fixed value of $\beta$, also suggests that $\lambda_{1}$ not only depends on the fragmentation as defined by $s$, the number of similar neighbours. This is again confirmed by the comparison between Figures 6 (b) and 8 (b); for fixed values of $s$ the value of $\lambda_{1}$ is, in most cases, larger in Figure 6 (b) than in Figure 8 (b). In particular, the persistence threshold for landscapes generated by model 1 was reached for values 
of $s$ around 4600 whereas it was reached around 4450 for the second landscape model. Comparing these two models, our interpretation is that $\lambda_{1}$, and thus species survival, also depends on a more "global" idea of fragmentation, taking the proximity of the habitat patches into account, in addition to the number of similar neighbours $s$.

The reader may note that our results were obtained for particular values of $\mu^{+}$and $\mu^{-}$. However, as emphasized at the end of section 2 the survival can easily be assessed for the whole family of growth rate functions $\{\mu(\mathbf{x})-\alpha\}_{\alpha \in \mathbb{R}}$, since from formula $(2.5), \lambda_{1}[\mu(\mathbf{x})-\alpha]=\lambda_{1}[\mu(\mathbf{x})]+\alpha$. The above formula also underlines the interest of having values of $\lambda_{1}$ as low as possible. Indeed, $\mu^{+}$and $\mu^{-}$being fixed, when subtracting $\alpha$ to the growth rate, the chances of species persistence are all the more important as $\lambda_{1}[\mu]$ was low before the change. Such a modification in the growth rate function could for instance occur as a result of a climate change. Thus, living in an aggregated habitat, and having a strongly negative value of $\lambda_{1}$, not only ensures the species survival but also gives to the modelled species more robustness to this kind of external perturbations.

In this paper, the habitat abundance was set to $a=20 \%$. For other values of the habitat abundance $a$, it would be natural to expect comparable results. However, some differences may appear since, for higher habitat abundance, and also depending on the value of the amplitude $\left|\mu^{+}-\mu^{-}\right|$, the optimal configuration can no longer be disc-shaped, but can for instance take the form of a stripe [31]. A slight modification of the function $\tau_{\kappa}$ in landscape model 2 would allow to converge to such configurations as $\beta$ increase. The analysis of the relative influence of habitat fragmentation and habitat abundance on species persistence is also an interesting issue. For instance, from formula (2.4) we know that $-\mu^{-}-\frac{a}{100}\left(\mu^{+}-\mu^{-}\right)$is an upper bound of $\lambda_{1}$. Thus, for $a=23 \%$, and with our values of $\mu^{+}, \mu^{-}$and $C$, we get $\lambda_{1} \leq-0.1$. This means that the most fragmented habitat configurations, with an abundance of $23 \%$, are equivalent to the most aggregated ones with a habitat abundance of $20 \%$ in terms of species persistence. This relative influence of fragmentation and abundance depends on the values of $D, \mu^{+}$and $\mu^{-}$. Further work is needed to fully understand it.

Another natural problem is whether similar results, linking habitat fragmentation and population persistence, can be obtained in a general (non-periodic) infinite environment. In this case a generalised principal eigenvalue $\lambda_{1}^{\prime}$ can be defined by $\lambda_{1}^{\prime}:=\inf \left\{\lambda \in \mathbb{R}\right.$, s. t. $\exists \phi>0 \in C^{2}\left(\mathbb{R}^{N}\right) \cap W^{1, \infty}\left(\mathbb{R}^{N}\right)$ with $\left(D \nabla^{2}+\mu(\mathbf{x})+\lambda\right) \phi \leq 0$ in $\left.\mathbb{R}^{N}\right\}$ (see [1] for the definition of $\left.W^{1, \infty}\left(\mathbb{R}^{N}\right)\right)$. As proved in [7], the existence of a positive stationary state of (2.2) is almost completely determined by the sign of $\lambda_{1}^{\prime}$ (except when $\lambda_{1}^{\prime}=0$; see also [14] for a probabilistic version of this result). However, although $\lambda_{1}^{\prime}>0$ is still a sufficient condition for population extinction, $\lambda_{1}^{\prime}<0$ may not always imply persistence, in the meaning of section 2. Another difficulty comes from the existence, with most landscape models, of very large habitat patches (as large as we want since the environment is infinite). Consequently, the species always survives, as soon as $\mu^{+}>0$, independently of the degree of fragmentation. Therefore, the study of the effects of habitat fragmentation on population persistence requires to set a constraint on the maximal habitat patches size. The periodicity of the 
environment is a particular case of such a constraint.

\section{Appendix A. Derivation of the Spearman's $\rho$}

For the readers that are not familiar with this notion, we recall here the definition of the Spearman's $\rho$ coefficient (see e.g. [35]).

Let $\beta_{1}, \ldots, \beta_{n c}$ be the values of $\beta$ for which computations were performed (with repetitions), and $\lambda_{1,1}, \ldots, \lambda_{1, n c}$ the corresponding values of $\lambda_{1}$. Let us define a vector $V_{1}$ in the following way: for each integer $i$ in $[1, n c]$, set $p_{i}=$ \{number of $k \mid \beta_{k}<\beta_{i}$, and $r_{i}=\left\{\right.$ number of $k \mid \beta_{k}=\beta_{i}$. We set $V_{1, i}=$ $\frac{\left(p_{i}+1\right)+\left(p_{i}+r_{i}\right)}{2}$. This corresponds to the rank of $\beta_{i}$ with medium value when $\beta_{i}$ is repeated. Similarly, we define a vector $V_{2}$ corresponding to the rank of $\lambda_{i}$. Let us define $D$ by $D_{i}=V_{2, i}-V_{1, i}$ for $i=1, \ldots, n c$. Then Spearman's $\rho_{s}$ is given by the following formula

$$
\rho_{s}=1-\frac{6 D \cdot D}{n c\left(n c^{2}-1\right)}
$$

\section{Acknowledgements}

We would like to thank Joël Chadœeuf and the three anonymous referees for their valuable suggestions and comments.

\section{References}

[1] Adams, R.A.: Sobolev Spaces. Academic Press, New-York, 1975

[2] Amann, H.: Fixed point equations and nonlinear eigenvalue problems in ordered Banach spaces. SIAM Rev. 18, 620-709 (1976)

[3] Baillie, J.E.M., Hilton-Taylor, C., Stuart, S.N., (eds): 2004 IUCN Red List of Threatened Species. A Global Species Assessment. IUCN, Gland, Switzerland and Cambridge, UK, 2004

[4] Berestycki, H., Hamel F.: Front propagation in periodic excitable media. Comm. Pure Appl. Math. 55, 949-1032 (2002)

[5] Berestycki, H., Hamel, F., Roques, L.: Analysis of the periodically fragmented environment model : I - Species persistence. J. Math. Biol. 51 (1), 75-113 (2005)

[6] Berestycki, H., Hamel, F., Roques, L.: Analysis of the periodically fragmented environment model : II - Biological invasions and pulsating travelling fronts. J. Math. Pures Appl. 84 (8), 1101-1146 (2005) 
[7] Berestycki, H., Hamel, F., Rossi, L.: Liouville type results for semilinear elliptic equations in unbounded domains. Annali Mat. Pura Appl., to appear (2007)

[8] Cantrell, R.S., Cosner, C.: Diffusive logistic equations with indefinite weights: population models in disrupted environments. Proc. Roy. Soc. Edinburgh 112, 293-318 (1989)

[9] Cantrell, R.S., Cosner, C.: Diffusive logistic equations with indefinite weights: population models in disrupted environments II. SIAM J. Math. Anal. 22 (4), 1043-1064 (1991)

[10] Cantrell, R.S., Cosner, C.: The effects of spatial heterogeneity in population dynamics. J. Math. Biol. 29, 315-338 (1991)

[11] Cantrell, R.S., Cosner, C.: Spatial Ecology via Reaction-Diffusion Equations. Series In: Mathematical and Computational Biology, John Wiley and Sons, Chichester, Sussex UK, 2003

[12] Chekroun, M.D., Roques, L.J.: Models of population dynamics under the influence of external perturbations: mathematical results. C. R. Acad. Sci. Paris, Ser. I 343, 307-310 (2006)

[13] Ciarlet, P.G.: Numerical Analysis of the Finite Element Method. Seminaire de Mathematiques Superieures 59, Les Presses de l'Universite de Montreal. Montreal, 1976

[14] Engländer, J., Kyprianou, A.E.: Local extinction versus local exponential growth for spatial branching processes. Ann. Probab. 32, 78-99 (2004)

[15] Fisher, R.A.: The advance of advantageous genes. Ann. Eugenics 7, 335369 (1937)

[16] Gardner, R.H., Milne, B.T., Turner, M.G., O’Neill, R.V.: Neutral Models for the Analysis of Broad-Scale Landscape Pattern. Landscape Ecol. 1 (1), 19-28 (1987)

[17] Gross, L.J., Rose, K.A., Rykiel, E.J., Van Winkle, W., Werner, EE.: Individual-based modeling. In: DeAngelis, D.L., Gross, L.J., (eds.), Summary of a workshop. Individual-based Models and Approaches in Ecology: Populations, Communities, and Ecosystems 511-552. Routledge, Chapman and Hall, New York, 1992

[18] Hastings, W.K.: Monte Carlo sampling methods using Markov Chains and their applications. Biometrika 57, 97-109 (1970)

[19] Hess, P.: Periodic-parabolic boundary value problems and positivity. Pitman Research Notes in Mathematics Series, 247. Longman Scientific \& Technical, Harlow, UK, 1991 
[20] Hutson, V., Mischaikow, K., Polacik, P.: The Evolution of dispersal rates in a heterogeneous time-periodic environment. J. Math. Biol. 43, 501-533 (2001)

[21] Kareiva, P.M., Shigesada, N.: Analyzing insect movement as a correlated random walk. Oecologia 56, 234-238 (1983)

[22] Keitt, T.H.: Spectral representation of neutral landscapes. Landscape Ecol. 15, 479-494 (2000)

[23] Kinezaki, N., Kawasaki, K., Takasu, F., Shigesada, N.: Modeling biological invasion into periodically fragmented environments. Theor. Popul. Biol. 64, 291-302 (2003)

[24] Kolmogorov, A.N., Petrovsky, I.G., Piskunov, N.S.: Étude de l'équation de la diffusion avec croissance de la quantité de matière et son application à un problème biologique. Bulletin Université d'État à Moscou (Bjul. Moskowskogo Gos. Univ.), Série internationale A 1, 1-26 (1937)

[25] Marsh, L.M., Jones, R.E.: The form and consequences of random walk movement models. J. Theor. Biol. 133, 113-131 (1988)

[26] Metropolis, N., Rosenbluth, A.W., Rosenbluth, N.M., Teller, A.H., Teller, E.: Equation of state calculations for fast computing machines. J. Chem. Phys. 21, 1087-1092 (1953)

[27] Murray, J.D.: Mathematical Biology. Second corrected edition, Springer Verlag, New York, 1993

[28] Okubo A., Levin, S.A.: Diffusion and Ecological Problems - Modern Perspectives. Second edition, Springer-Verlag, New-York, 2002

[29] Palmer, M.W.: The coexistence of species in fractal landscapes. Am. Nat. 139, 375-397 (1992)

[30] Robert, C.P., Casella, G.: Monte Carlo statistical methods. SpringerVerlag, New York, 1999

[31] Roques, L., Hamel, F.: On optimal habitat configurations for species persistence. INRA research report RR2006_25 (2006)

[32] Saunders, D.A., Hobbs, R.J., Margules, C.R.: Biological consequences of ecosystem fragmentation: a review. Conserv. Biol. 51, 18-32 (1991)

[33] Shigesada N., Kawasaki K.: Biological invasions: theory and practice. Oxford Series in Ecology and Evolution, Oxford: Oxford University Press, 1997

[34] Shigesada, N., Kawasaki, K., Teramoto, E.: Traveling periodic waves in heterogeneous environments. Theor. Popul. Biol. 30, 143-160 (1986) 
[35] Sprent, P.: Applied Nonparametric Statistical Methods. Second edition. Chapman and Hall, London, 1993

[36] Stoyan, D., Kendall, W.S., Mecke, J.: Stochastic Geometry and its Applications. Second edition, Wiley Series in Probability and Statistics, John Wiley \& Sons, Chichester, 1995

[37] Turchin, P.: Quantitative Analysis of Movement: Measuring and Modeling Population Redistribution in Animals and Plants. Sinauer Associates, Sunderland, MA, 1998

[38] With, K.A., King, A.W.: Extinction thresholds for species in fractal landscapes. Conserv. Biol. 13, 314-326 (1999) 